\title{
UTILIZAÇÃO DE FÓSFORO DO SOLO E DO FERTILIZANTE POR TOMATEIRO
}

\author{
Felipe Carlos Alvarez V.1,2,5,6*; Robson Rui Cotrim Duete ${ }^{1,2,4}$; Takashi Muraoka²; Washington \\ Luiz Cotrim Duete ${ }^{3}$; Cassio Hamilton Abreu Jr. ${ }^{2}$ \\ ${ }_{2}^{1}$ Pós-Graduando em Energia Nuclear na Agricultura - USP/CENA. \\ ${ }^{2}$ Lab. de Fertilidade do Solo - USP/CENA, C.P. 96 - CEP: 13400-970 - Piracicaba, SP. \\ ${ }^{3}$ Depto. de Química Agrícola e Solos - UFBA/EA - CEP: 44380-000 - Cruz das Almas, BA. \\ ${ }_{5}^{4}$ Empresa Baiana de Desenvolvimento Agrícola S/A. \\ "Instituto de Investigaciones Fundamentales en Agricultura Tropical "Alejandro de Humboldt" - CEP: 17200 - Santiago \\ de las Vegas, Ciudad Habana, Cuba. \\ ${ }^{6}$ Bolsista CNPq. \\ *Autor correspondente<falvarez@cena.usp.br>
}

\begin{abstract}
RESUMO: O fósforo, embora seja o macronutriente em menor concentração na maioria das culturas, é o mais aplicado como fertilizante mineral, constituindo uma parcela significativa do custo de produção agrícola. O conhecimento das diferenças entre variedades de plantas em relação à absorção do fósforo do solo e do fertilizante torna-se de grande importância para programas de melhoramento e manejo da adubação fosfatada. Utilizando a técnica do traçador radioativo ${ }^{32} \mathrm{P}$, foram avaliadas as diferenças na absorção de fósforo proveniente do solo e do fertilizante (superfosfato triplo), na disponibilidade de $\mathrm{P}$ do solo pelos valores $L$ e $A$ e nas eficiências fisiológica, ecofisiológica e agronômica do nutriente pelas variedades comerciais de tomate (Licopersicum esculentum Will) Santa Clara e Santa Cruz. Conduziu-se um experimento em condições de casa de vegetação com dois solos (Latossolo Vermelho distrófico e Latossolo Vermelho-Amarelo eutrófico) e dois níveis de $\mathrm{P}\left(0\right.$ e $\left.31,1 \mathrm{mg} \mathrm{kg}^{-1}\right)$. A resposta das plantas foi diferente nos dois solos e em concordância com suas características. Houve aumentos significativos, em ambos os solos, na produção de matéria seca, no valor $L$ e na absorção de $\mathrm{P}$ do solo devido à aplicação do fertilizante mineral, mas não no valor $A$. Diferenças entre variedades quanto à produção de matéria seca, concentração de fósforo, eficiência fisiológica, ecofisiológica e agronômica foram mais marcadas no solo Latossolo Vermelho-Amarelo eutrófico. Não houve diferenças entre as variedades na absorção de $\mathrm{P}$ do solo e do fertilizante, valores $L$ e $A$.
\end{abstract} Palavras-chave: Licopersicum esculentum, valor $A$, valor $L,{ }^{32} \mathrm{P}$, radioisótopo

\section{SOIL AND FERTILIZER PHOSPHORUS USE BY TOMATO}

\begin{abstract}
Phosphorus, despite its relatively low content in most crops is the most applied nutrient as mineral fertilizer and is an important fraction of the cost of agricultural production. Knowledge of the differences among plant varieties in relation to soil and fertilizer $\mathrm{P}$ uptake is fundamental for breeding improvement programs and management of phosphate fertilization. Differences in $\mathrm{P}$ uptake from soil and fertilizer (triple superphosphate), soil $\mathrm{P}$ availability through $L$ and $A$ values, and the physiologic, ecophysiologic and agronomic efficiencies of two commercial tomato (Lycopersicum esculentum Will) varieties, Santa Clara and Santa Cruz, were evaluated by the ${ }^{32} \mathrm{P}$ technique. The experiment was carried out in a greenhouse, using two soils, a dystrophic Dark Red Latosol and an eutrophic Red Yellow Latosol, and two P fertilizer application rates ( 0 and $31.1 \mathrm{mg} \mathrm{kg}^{-1}$ ). Plant responses were markedly different in the two different soils. There was a clear increase in dry matter production, $L$ value, and $\mathrm{P}$ uptake from the soil due to fertilizer application, but not in the $A$ values. Differences in dry matter production, $\mathrm{P}$ concentration and physiologic, ecophysiologic and agronomic efficiency between varieties differed more for the eutrophic Red Yellow Latosol. There was no difference between the two varieties in relation to the uptake of soil and fertilizer $P, L$ and $A$ values.
\end{abstract}

Key words: $L$ icopersicum esculentum, $A$ value, $L$ value, ${ }^{32} \mathrm{P}$, radioisotope

\section{INTRODUÇÃO}

As maiores limitações na produção agrícola, em solos ácidos de regiões tropicais e subtropicais, são a baixa disponibilidade de fósforo no solo, dada a alta capacidade de adsorção e ou baixo teor do nutriente no material de origem, e a baixa eficiência de absorção e utilização do fósforo apresentada pela maioria das variedades modernas empregadas comercialmente (Novais \& Smyth, 1999). Essas condições têm exigido a aplicação de elevadas doses de fertilizante fosfatado. Em decorrência destes fatos, tem sido sugerido o uso de cultivares eficientes na absorção e utilização de nutrientes em condições de baixa disponibilidade de fósforo (Silva \& Gabelman, 1992), em conjunto com práticas que melhorem a fertilidade, disponibilidade de fósforo e de água do solo para as plantas (adubação fosfatada localizada, rotação de culturas, plantio direto, etc.), objetivando prolongar a vida útil das reservas naturais de fosfato e reduzir o custo de produção, dois grandes interesses para melhoristas, produtores e consumidores. 
As metodologias com ${ }^{32} \mathrm{P}$ têm sido amplamente usadas na determinação do fósforo disponível do solo (valores $A, L$ e $E$ ) e na diferenciação do fósforo na planta proveniente do solo e do fertilizante (Neptune \& Muraoka, 1978; Fardeau et al., 1996). Os valores $L$ e $A$ independem da análise de $\mathrm{P}$ do solo; porém são dependentes das condições experimentais (ex.: espécie vegetal, estádio fisiológico da planta, interação entre nutrientes, matéria orgânica, volume e umidade do solo, etc.) (Volk \& McLean, 1963; Neptune \& Muroka, 1978; Morales, 1981).

Se as espécies vegetais têm capacidades distintas em absorver o $\mathrm{P}$ do solo, é de se esperar valores $L$ e $A$ diferentes, possibilitando a utilização dessas variáveis para distinguir genótipos com maior eficiência na absorção de $P$ do solo ou tolerância a baixos teores do nutriente e, em trabalho futuros, para seleção de variedades úteis ao desenvolvimento de uma agricultura sustentável.

O tomateiro é a hortaliça mais importante no Brasil tanto em termos de produção como em valor econômico, com 56 mil hectares dedicados a essa cultura (FNP Consultoria e Comércio, 2000), consumindo grande quantidade de fertilizante fosfatado em face da área cultivada, da exigência pela cultura e da alta adsorção do nutriente no solo.

A ocorrência de variabilidade genotípica quanto à tolerância ao baixo teor de fósforo tem sido relatada em várias culturas de interesse econômico (Silva \& Gabelman, 1992; Horts \& Wiesler, 1996), inclusive no tomateiro (Coltman et al., 1985). No Brasil, inúmeros trabalhos foram conduzidos para avaliar as características morfológicas, agronômicas, qualidade de frutos e adaptação edafoclimática de cultivares de tomate, das quais as variedades Santa Cruz e Santa Clara configuram como progenitoras de importantes genótipos (Leal, 1973; Silva, 1996; Peixoto et al., 1999), porém, são muito escassas as informações quanto à nutrição e eficiência de utilização do fósforo pelo tomateiro.

O presente trabalho teve por objetivo avaliar as diferenças na absorção de fósforo proveniente do solo e do fertilizante, na disponibilidade de $\mathrm{P}$ do solo pelos valores $L$ e $A$ e nas eficiências fisiológica, ecofisiológica e agronômica do nutriente pelas variedades de tomate Santa Clara e Santa Cruz, em dois solos com propriedades químicas diferentes.

\section{MATERIAL E MÉTODOS}

O experimento foi conduzido em condições de casa de vegetação no Centro de Energia Nuclear na Agricultura (USP/CENA), em Piracicaba (SP). Os tratamentos foram constituídos de dois solos: um Latossolo Vermelho distrófico (LV) da região de Piracicaba, SP, e um Latossolo Vermelho-Amarelo eutrófico (LVA) da região de Irecê, BA; de duas variedades de tomate: Santa Cruz e Santa Clara; e de duas doses de fósforo: 0 e $31,1 \mathrm{mg} \mathrm{kg}^{-1}$. As unidades experimentais foram constituídas por vasos plásticos contendo $2,5 \mathrm{~kg}$ de amostra dos solos e o delineamento experimental utilizado foi em blocos ao acaso, com arranjo fatorial para solos, variedades e níveis de $\mathrm{P}, \mathrm{com}$ quatro repetições.

As amostras de ambos os solos foram coletadas na camada $0-20 \mathrm{~cm}$ de profundidade, secas ao ar, destorroadas e passadas em peneira de $5 \mathrm{~mm}$ de malha. Três subamostras dos solos foram passadas por peneira de $2 \mathrm{~mm}$ de malha e caracterizadas química e fisicamente (Tabela 1). As análises químicas foram feitas de acordo com o método descrito em Raij et al. (1987) e as físicas pelo método do densímetro, conforme metodologia adotada pelo Departamento de Solos e Nutrição de Plantas da ESALQ.

Ambos os solos foram considerados de textura argilosa. O solo LV apresentou reação ácida, com presença de alumínio trocável e o LVA, ligeiramente alcalina, sem alumínio trocável, mas com presença de carbonato de cálcio livre. A soma de bases foi alta no

Tabela 1 - Características químicas e físicas dos solos utilizados (camada de $0-20 \mathrm{~cm}$ ).

\begin{tabular}{|c|c|c|}
\hline \multirow{2}{*}{ Característica química e física } & \multicolumn{2}{|c|}{ Solo } \\
\hline & LVA & LV \\
\hline pH em água $(1: 2,5)$ & 7,90 & 4,68 \\
\hline pH em KCl $(1: 2,5)$ & 6,90 & 3,94 \\
\hline$\Delta \mathrm{pH}$ & $-1,00$ & $-0,74$ \\
\hline M.O. $\left(\mathrm{g} \mathrm{dm}^{-3}\right)$ & 25,00 & 26,00 \\
\hline P-resina $\left(\mathrm{mg} \mathrm{dm}^{-3}\right)$ & 46,00 & 12,05 \\
\hline P-total $\left(\mathrm{mg} \mathrm{dm}^{-3}\right)$ & 606,80 & 497,60 \\
\hline 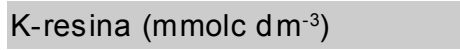 & 17,00 & 0,21 \\
\hline Na-Mehlich (mmolc d m$\left.{ }^{-3}\right)$ & 1,10 & 1,40 \\
\hline Ca-resina $\left({\left.\mathrm{mmolc} \mathrm{dm}^{-3}\right)}\right.$ & 84,70 & 11,77 \\
\hline Mg-resina $\left(\mathrm{mmolc} \mathrm{dm}^{-3}\right)$ & 26,00 & 1,73 \\
\hline $\mathrm{Al}^{3+}\left(\mathrm{mmolc} \mathrm{dm}^{-3}\right)$ & 0,00 & 1,08 \\
\hline $\mathrm{H}^{+}+\mathrm{Al}^{3+}\left(\mathrm{mmolc} \mathrm{dm}^{-3}\right)$ & 12,00 & 45,68 \\
\hline $\mathrm{CaCO}_{3}$ (equivalente \%) & 0,99 & 0,00 \\
\hline SB $\left(\mathrm{mmolc} \mathrm{dm}^{-3}\right)^{1}$ & 128,80 & 15,11 \\
\hline CTC $\left(\mathrm{mmolc} \mathrm{dm}^{-3}\right)^{2}$ & 140,80 & 60,79 \\
\hline Saturação por bases $(\mathrm{V} \%)^{3}$ & 91,48 & 24,86 \\
\hline $\mathrm{Al}_{2} \mathrm{O}_{3}(\%)$ & 12,00 & 10,00 \\
\hline $\mathrm{Fe}_{2} \mathrm{O}_{3}(\%)$ & 4,60 & 6,54 \\
\hline Areia $\left(\mathrm{g} \mathrm{kg}^{-1}\right)$ & 270 & 270 \\
\hline Silte $\left(\mathrm{g} \mathrm{kg}^{-1}\right)$ & 220 & 172 \\
\hline Argila $\left(\mathrm{g} \mathrm{kg}^{-1}\right)$ & 510 & 637 \\
\hline Adsorção de $P\left(\mathrm{~g} \mathrm{~kg}^{-1}\right)$ & 871 & 1359 \\
\hline Dessorção de $P\left(\mathrm{~g} \mathrm{~kg}^{-1}\right)$ & 148 & 140 \\
\hline Dessorção relativa de $\mathrm{P}(\%)$ & 17 & 10 \\
\hline
\end{tabular}

${ }^{1} \mathrm{SB}$ (soma de bases) $=\mathrm{Ca}^{2+}+\mathrm{Mg}^{2+}+\mathrm{K}^{+}+\mathrm{Na}^{+}$.

${ }^{2} \mathrm{CTC}$ (capacidade de troca catiônica) $=\mathrm{SB}+\left(\mathrm{H}^{+}+\mathrm{Al}^{3+}\right)$.

${ }^{3} \mathrm{~V} \%=\left(\mathrm{SB}^{*} 100\right) / \mathrm{CTC}$. 
LVA e baixa no LV. O teor de matéria orgânica foi médio em ambos os solos. O teor de $\mathrm{P}$ extraído pela resina foi alto no solo LVA e baixo no LV (Tabela 1).

Visando facilitar a interpretação do comportamento das variedades de tomate quanto à nutrição fosfatada nos solos utilizados, foram também determinadas as capacidades de adsorção e dessorção de P (Tabela 1) e as frações de $P$ inorgânico (Tabela 2) antes e depois da adsorção do $P$ (Rachinskii, 1974). Para a adsorção de $\mathrm{P}, 2 \mathrm{~g}$ de amostras de solo foram mantidas em agitação por 48 horas com $20 \mathrm{~mL}$ de $\mathrm{CaCl}_{2}$ 0,01 mol mL ${ }^{-1}$, contendo $0,25 \mathrm{mg} \mathrm{mL}^{-1}$ de $\mathrm{P}$ na forma de $\mathrm{KH}_{2} \mathrm{PO}_{4}$ marcado com $1,85 \mathrm{~Bq} \mathrm{~mL}^{-1}$ de ${ }^{32} \mathrm{P}$ $\left(\mathrm{KH}_{2}{ }^{32} \mathrm{PO}_{4}\right.$ livre de carregador). A adsorção do $\mathrm{P}$ foi calculada pela relação de radioatividades do ${ }^{32} \mathrm{P}$ de volumes iguais da solução antes e depois do contato com a amostra de solo. Para a dessorção, a mesma amostra de solo empregada para a adsorção foi lavada duas vezes com $10 \mathrm{~mL}$ de álcool e agitada por $24 \mathrm{~h}$ com $20 \mathrm{~mL}$ de $\mathrm{CaCl}_{2}$ de $0,01 \mathrm{~mol} \mathrm{~mL}^{-1}$. A relação de radioatividades em volumes iguais desta solução e daquela inicialmente utilizada para a adsorção, foi utilizada para o cálculo da dessorção de P.

A determinação das frações do $P$ inorgânico antes e depois da adsorção, no solo LV, foi baseada na solubilização seletiva dos compostos de fósforo nas soluções de $\mathrm{NH}_{4} \mathrm{Cl} 1 \mathrm{~mol} \mathrm{~L}^{-1} ; \mathrm{NH}_{4} \mathrm{~F} 0,5 \mathrm{~mol} \mathrm{~L}^{-1}$ a pH 7,0;

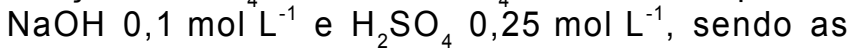
frações de $\mathrm{P}$ extraídas denominadas, respectivamente, de $\mathrm{P}$ solúvel, ligado ao alumínio (P-Al), ligado ao ferro (P-Fe) e ligado ao cálcio ( $\mathrm{P}-\mathrm{Ca}$ ) (Olsen \& Sommers, 1982).

Para o solo LVA foi aplicada a modificação proposta por Smillie \& Syers, (1972). Esta modificação consistiu em realizar, após a extração com $\mathrm{NH}_{4} \mathrm{Cl}$ $1 \mathrm{~mol} \mathrm{~L}^{-1}$, uma extração com $\mathrm{NaOH} 0,1 \mathrm{~mol} \mathrm{~L}^{-1}$ para obtenção da fração P-Fe + P-Al, e uma terceira extração com $\mathrm{HCl} 0,5 \mathrm{~mol} \mathrm{~L}^{-1}$ para a fração P-Ca. Todas as frações de $P$ inorgânico foram obtidas nas relações solo:solução de 1:50 (m/v). A determinação de $P$ nos extratos foi feita por colorimetria conforme descrito em Raij et al. (1987), com curva padrão específica para cada extrato.
Para a condução do experimento com plantas, adicionaram-se $100 \mathrm{~mL}$ de solução $\mathrm{KH}_{2}{ }^{32} \mathrm{PO}_{4}$, contendo $3,7 \mathrm{MBq}$ de ${ }^{32} \mathrm{P}$ livre de carregador, a cada amostra de solo contida nos vasos. Dois dias após, foi aplicado fertilizante fosfórico na forma de superfosfato triplo e homogeneizada a amostra de solo. Em cada vaso, foram colocadas seis sementes de tomate da variedade correspondente a cada tratamento e aplicada água suficiente para elevar a umidade a cerca de $70 \%$ da capacidade máxima de retenção de água, repondo-se as perdas através de irrigações diárias com água destilada. Cinco dias após a germinação, realizou-se desbaste, mantendo três plantas por vaso. Nesta ocasião, foram aplicados nitrogênio e potássio, nas formas de uréia e $\mathrm{KCl}$ em solução, ambos nutrientes nas doses de $50 \mathrm{mg} \mathrm{kg}^{-1}$, respectivamente, em todos os tratamentos.

Aos quarenta e cinco dias após a germinação, a parte aérea das plantas foi coletada, lavada, secada em estufa a $60-70{ }^{\circ} \mathrm{C}$ e moída após a obtenção do peso de matéria seca. $\mathrm{O}$ material vegetal foi submetido à digestão nítrico-perclórica para a determinação do teor de $\mathrm{P}$, pelo método colorimétrico metavanadato-molíbdico (Jackson, 1958).

A radioatividade do ${ }^{32} \mathrm{P}$, nos extratos de solo e de planta, foi medida por efeito Cerenkov em contador de cintilação líquida (Wallac 1409). Determinou-se a atividade específica em desintegrações por minuto por unidade de $\mathrm{P}\left(\mathrm{dpm} \mathrm{mg} \mathrm{m}^{-1}\right)$ no material vegetal e estimadas as porcentagens de $\mathrm{P}$ na planta proveniente do solo (\%Ppps) e do fertilizante (\%Pppf). O valor $A$ foi obtido pelo método indireto de marcação do solo $\mathrm{com}^{32} \mathrm{P}$ e o valor $L$, com a equação "sem carregador de fósforo" nos tratamentos que não foram adubados e com a equação "com carregador de fósforo" nos tratamentos adubados, conforme descrito em Neptune \& Muraoka (1978) e Fardeau et al. (1996).

A eficiência de utilização do $P$ pelas plantas de tomate foi analisada por aspectos ecofisiológicos, fisiológicos e agronômicos (Bowen \& Zapata, 1991). O ecofisiológico define a eficiência em relação da quantidade do nutriente absorvido pela planta com àquela que foi

Tabela 2 - Fracionamento do fósforo inorgânico nas amostras de solos empregadas no experimento.

\begin{tabular}{|c|c|c|c|c|c|c|}
\hline \multirow[b]{2}{*}{ Solo } & & \multicolumn{3}{|c|}{$\begin{array}{l}\text { Fração de fósforo inorgânico na amostra do solo antes e após } \\
\text { a adsorção de } P \text { e respectivo desvio padrão }\end{array}$} & \multicolumn{2}{|c|}{$\begin{array}{l}\text { Porcentagem relativa da fração em } \\
\text { relação à } \mathrm{P}-\mathrm{Fe}+\mathrm{P}-\mathrm{Al}+\mathrm{P}-\mathrm{Ca}\end{array}$} \\
\hline & & $\begin{array}{l}\text { P solúvel em } \\
\mathrm{NH}_{4} \mathrm{CL}\end{array}$ & $\begin{array}{l}\text { Fósforo ligado ao } \\
\text { ferro e alumínio } \\
\text { (P-Fe + P-Al) }\end{array}$ & $\begin{array}{c}\text { Fósforo ligado ao } \\
\text { cálcio } \\
(\mathrm{P}-\mathrm{Ca})\end{array}$ & $\mathrm{P}-\mathrm{Fe}+\mathrm{P}-\mathrm{Al}$ & $\mathrm{P}-\mathrm{Ca}$ \\
\hline \multirow{3}{*}{ LVA } & & - & $\mathrm{mg} \mathrm{dm}^{-3}$ & - & ------------------ & ------------ \\
\hline & 1 & ND & $70,8 \pm 0,9$ & $37,0 \pm 0,9$ & 65,7 & 34,3 \\
\hline & 2 & $566,7 \pm 8,3$ & $185,4 \pm 10,9$ & $381,3 \pm 3,8$ & 32,6 & 67,4 \\
\hline \multirow{2}{*}{ LV } & 1 & ND & $118,6 \pm 2,1$ & $12,3 \pm 0,3$ & 90,6 & 9,4 \\
\hline & 2 & $120,3 \pm 1,9$ & $655,3 \pm 16,1$ & $25,8 \pm 1,3$ & 96,2 & 3,8 \\
\hline
\end{tabular}

1 = antes da adsorção e 2 = após adsorção de $\mathrm{P}$.

ND = não detectado. 
aplicada como fertilizante ( $\mathrm{g}$ de $\mathrm{P}$ acumulado por $\mathrm{kg}$ de $\mathrm{P}$ aplicado). O fisiológico define a eficiência com que o nutriente absorvido pela planta foi utilizado para produzir o rendimento, no caso, matéria seca (kg de MS por $\mathrm{g}$ de $P$ acumulado). $O$ agronômico define a eficiência de utilização do nutriente aplicado $(\mathrm{kg}$ MS por $\mathrm{kg}$ de $\mathrm{P}$ aplicado). As eficiências ecofisiológica e agronômica foram calculadas em relação ao $\mathrm{P}$ disponível dos solos para os tratamentos sem adição de $\mathrm{P}$ e, em relação à somatória do $\mathrm{P}$ disponível do solo e o aplicado, para os tratamentos fertilizados, levando em consideração que ambas as fontes do nutriente são utilizadas pelas plantas.

Os dados obtidos foram submetidos às análises de variância e as médias comparadas pelo teste de Tukey ao nível de significância de $5 \%$. As análises exploratória e estatística dos dados foram realizadas pelo programa estatístico SAS lab/SAS.

\section{RESULTADOS E DISCUSSÃO}

A produção de matéria seca pelas cultivares de tomateiro foi superior no solo LVA (Tabela 3). Neste solo a variedade Santa Cruz foi superior à variedade Santa Clara na produção de matéria seca, tanto na presença quanto na ausência do adubo fosfatado. Diferenças entre variedades, quanto à produção de matéria seca e nutrição fosfórica, também foram relatadas por Coltman et al. (1985) em tomateiro, por Silva \& Gabelman (1992) em milho e por Horts \& Wiesler (1996) em trigo.

Os teores de $\mathrm{P}$ na parte aérea foram maiores na Santa Clara no solo LVA na presença e ausência de fertilizante fosfatado, respectivamente (Tabela 3), porém, não houve diferenças entre variedades quanto à quantidade total de $P$ acumulado na parte aérea das plantas.

No solo LV (Tabela 3), embora não tenha havido diferença estatística na produção de matéria seca entre as variedades no tratamento com fertilizante, mantevese a superioridade da variedade Santa Cruz no tratamento testemunha. Os teores de $P$ na planta não foram diferentes entre variedades e o acúmulo de $\mathrm{P}$ na parte aérea só apresentou diferença no tratamento testemunha, sendo maior na variedade Santa Cruz. A produção de matéria seca e o acúmulo de $\mathrm{P}$ foram muito inferiores aos observados no solo LVA. Esse comportamento está diretamente relacionado com o pH ácido associado a baixa fertilidade, alta capacidade de sorção e baixa dessorção de P do solo LV (Tabela 1), que afetam o desenvolvimento e nutrição das plantas mesmo com adubação N-P-K (Novais \& Smyth, 1999). De fato, a porcentagem de utilização do fertilizante, que não diferiu entre variedades dentro de cada solo, no solo LV foi dez vezes inferior àquela do solo LVA (Tabela 3 ).

A máxima absorção de íons ocorre entre valores de $\mathrm{pH} 5$ a 7 , enquanto que abaixo de 5 , é sensivelmente reduzida. Pombo \& Smith (1986) relataram baixo rendimento de matéria seca, devido à baixa absorção de
$\mathrm{P}$, em tomateiro cultivado em solo com $\mathrm{pH} 5,5$. Além disso, a presença de $\mathrm{Al}^{3+}$ no solo (Tabela 1 ) deve ter causado toxicidade às plantas de tomate em face da redução do crescimento, acúmulo de $P$ nas raízes $\mathrm{e}$ inibição do transporte para o caule e folhas, de modo semelhante ao verificado por Simon et al. (1994).

$\mathrm{O}$ acúmulo de igual quantidade de fósforo pelas duas variedades dentro de cada tipo de tratamento (testemunha e adubado) no solo LVA (Tabela 2) e uma maior produção de matéria seca pela variedade Santa Cruz com o menor teor de P, exceto no solo LV onde o acúmulo foi maior na Santa Cruz que Santa Clara, proporcionaram maiores valores de eficiência fisiológica na variedade Santa Cruz nas duas doses de $\mathrm{P}$ avaliadas (Tabela 3). Ressalta-se, todavia, que no solo LV a eficiência fisiológica mostrou um comportamento diferenciado, sendo o maior valor no tratamento não fertilizado para a variedade Santa Clara e sem diferença entre as variedades no tratamento fertilizado.

Em ambos os solos, os maiores valores na eficiência fisiológica de fósforo foram obtidos nos tratamentos em que não se aplicou fósforo. Tal fato concorda com a literatura (Silva \& Braga, 1993) e demonstra que as plantas, em geral, utilizam com maior eficiência o fósforo quando é menor a disponibilidade, expressando assim a maior potencialidade na utilização do nutriente absorvido.

A eficiência ecofisiológica de utilização do $P$ foi maior com o fornecimento de $P$ para as duas variedades no solo LVA e para a variedade Santa Clara no solo LV (Tabela 3). Observou-se diferença significativa entre as variedades no tratamento não adubado em ambos os solos. Desse modo, sob baixa disponibilidade de P, em relação ao tratamento adubado, a variedade Santa Cruz mostrou maior eficiência ecofisiológica na absorção do $P$ disponível do solo, embora não tenha existido diferença no acúmulo de $P$ entre as duas variedades, nesse tratamento. Já no tratamento adubado, a eficiência ecofisiológica não diferiu entre variedades.

A eficiência agronômica, que expressa a utilização do $\mathrm{P}$ do solo, adubado ou não, na produção de matéria seca, foi crescente com a adubação, em ambas as variedades, no solo LVA, e na Santa Clara, no solo LV. Novamente, a variedade Santa Cruz teve significativamente maior eficiência agronômica, com uma maior produção de matéria seca por unidade de $\mathrm{P}$ disponível no solo (Tabela 3).

A recuperação de $P$ do fertilizante, calculada pela relação entre quantidade de $\mathrm{P}$ na planta proveniente do fertilizante e a quantidade de $\mathrm{P}$ aplicada com adubo, foi baixa e não diferiu entre variedades (Tabela 3 ). Isso indica que o nutriente do fertilizante teria assumido formas menos disponíveis às plantas, segundo pode ser verificado pelos dados de adsorção e as transformações do $\mathrm{P}$ adsorvido nos dois solos (Tabelas 1 e 2).

Dados os fenômenos de troca e diluição isotópica entre $\mathrm{O}{ }^{32} \mathrm{P}$ adicionado e $\mathrm{O}{ }^{31} \mathrm{P}$-lábil do solo, a 
Tabela 3 - Índices agronômicos e isotópicos obtidos para tomateiros das variedades Santa Cruz e Santa Clara.

\begin{tabular}{|c|c|c|c|c|c|c|c|c|c|c|c|}
\hline Tratamento & $\begin{array}{c}\text { Matéria } \\
\text { seca }\end{array}$ & $\begin{array}{l}\text { Fósforo } \\
\text { acumulado }\end{array}$ & Teor de fósforo & $\begin{array}{l}\text { Eficiência } \\
\text { fisiológica }\end{array}$ & $\begin{array}{c}\text { Eficiência } \\
\text { agronômica }\end{array}$ & $\begin{array}{l}\text { Eficiência } \\
\text { ecofisiológica }\end{array}$ & valor $\mathrm{L}$ & $\mathrm{Ppps}^{(1)}$ & $P p p f^{(2)}$ & $\begin{array}{c}\text { Utilização } \\
\text { do } \\
\text { fertilizante }\end{array}$ & valor $\mathrm{A}$ \\
\hline & $\begin{array}{l}\text { g por } \\
\text { vaso }\end{array}$ & $\begin{array}{l}\text { mg por } \\
\text { vaso }\end{array}$ & $\begin{array}{l}\mathrm{g} \text { de } P \text { por } \\
\mathrm{kg} \text { de MS }\end{array}$ & $\begin{array}{l}\mathrm{kg} \text { de } \mathrm{MS} \\
\text { por } \mathrm{g} \text { de } \mathrm{P} \\
\text { acumulado }\end{array}$ & $\begin{array}{l}\mathrm{kg} \text { de } \mathrm{MS} \\
\text { por kg de } \\
\mathrm{P} \text { aplicado }\end{array}$ & $\begin{array}{c}\text { g de P } \\
\text { acumulado } \\
\text { por kg de P } \\
\text { aplicado }\end{array}$ & $\mathrm{mg} \mathrm{kg}^{-1}$ & ------ & $----\%$ & 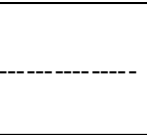 & $\mathrm{mg} \mathrm{kg}^{-1}$ \\
\hline \multicolumn{12}{|c|}{ LVA $(\mathrm{pH}$ água $=7,9)$} \\
\hline Sta. Cruz-0 & $1,48 c^{\#}$ & $2,01 \mathrm{~b}$ & $1,37 \mathrm{c}$ & $0,73 \mathrm{a}$ & $12,9 \mathrm{c}(3)$ & $17,5 \mathrm{~b}$ & $113 b$ & - & - & - & - \\
\hline Sta. Cruz-1 & $4,83 a$ & $8,75 a$ & $1,80 \mathrm{~b}$ & $0,56 \mathrm{~b}$ & $25,1 \mathrm{a}$ & $45,4 \mathrm{a}$ & $600 a$ & $27,8 \mathrm{a}$ & $72,2 \mathrm{a}$ & 8,13 a & $12 \mathrm{a}$ \\
\hline Sta. Clara-0 & $0,65 d$ & $1,16 b$ & $1,76 \mathrm{~b}$ & $0,57 \mathrm{~b}$ & $5,6 \mathrm{~d}$ & $10,1 \mathrm{c}$ & $239 b$ & - & - & - & - \\
\hline Sta. Clara-1 & $3,75 b$ & $8,79 \mathrm{a}$ & $2,34 \mathrm{a}$ & $0,43 c$ & $19,5 b$ & $45,6 \mathrm{a}$ & 789 a & $36,6 \mathrm{a}$ & $63,4 \mathrm{a}$ & $7,17 \mathrm{a}$ & $18 \mathrm{a}$ \\
\hline \multicolumn{12}{|c|}{$\mathrm{LV}(\mathrm{pH}$ água $=4,7)$} \\
\hline Sta. Cruz-0 & $0,22 \mathrm{~B}$ & $0,31 \mathrm{~B}$ & $1,46 \mathrm{BC}$ & $0,68 \mathrm{~B}$ & $7,3 \mathrm{~A}$ & $10,3 \mathrm{~A}$ & $100 \mathrm{~B}$ & - & - & - & - \\
\hline Sta. Cruz-1 & $0,67 \mathrm{~A}$ & $1,05 \mathrm{~A}$ & $1,60 \mathrm{AB}$ & $0,63 \mathrm{BC}$ & $6,2 \mathrm{~A}$ & $9,7 \mathrm{~A}$ & $304 \mathrm{~A}$ & $47,2 \mathrm{~A}$ & $52,8 \mathrm{~A}$ & $0,71 \mathrm{~A}$ & $29 \mathrm{~A}$ \\
\hline Sta. Clara-0 & $0,08 \mathrm{C}$ & $0,10 \mathrm{C}$ & $1,26 \mathrm{C}$ & $0,79 \mathrm{~A}$ & $2,7 \mathrm{~B}$ & $3,3 \mathrm{~B}$ & $51 \mathrm{~B}$ & - & - & - & - \\
\hline Sta. Clara-1 & $0,57 \mathrm{~A}$ & $0,97 \mathrm{~A}$ & $1,71 \mathrm{~A}$ & $0,58 \mathrm{C}$ & $5,3 \mathrm{~A}$ & $9,0 \mathrm{~A}$ & $278 \mathrm{~A}$ & $36,2 \mathrm{~A}$ & $63,8 \mathrm{~A}$ & $0,79 \mathrm{~A}$ & $18 \mathrm{~A}$ \\
\hline
\end{tabular}

0 = sem adubo fosfatado e 1 = com adubo fosfatado.

\#Médias nas colunas, dentro de cada solo, seguidas de mesmas letras não diferem pelo teste de Tukey a $5 \%$.

(1) P na planta proveniente do solo.

(2) P na planta proveniente do fertilizante.

(3)Valor obtido em relação ao $\mathrm{P}$ disponível no solo (P resina) quando sem adubo fosfatado.

disponibilidade (fator quantidade) de $\mathrm{P}$ do solo pode ser avaliada pelo valor $L$ e pelo valor $A$, respectivamente, conforme descrito por Neptune \& Muraoka (1978), e Novais \& Smyth (1999). O valor $L$ (de "Labile", Fardeau et al., 1996) é a quantidade de fósforo disponível que é isotopicamente trocável e determinado pela relação de radioatividade específica na planta e na solução do solo, portanto, de natureza físico-químico-biológica; e o valor $A$ (de "Available") é a quantidade de fósforo disponível no solo em unidades correspondentes a uma fonte padrão de fertilizante fosfatado (Muraoka, 1991).

As variáveis isotópicas, medidas com o uso do ${ }^{32} \mathrm{P}$, valor $L$, valor $A$, \%Ppps e \%Pppf não diferiram entre as variedades testadas, dentro de cada solo (Tabela 3 ). Esse comportamento foi devido ao fato de que as variedades provavelmente apresentaram o mesmo padrão de absorção do nutriente nas condições experimentais testadas. Todavia, foram observados aumentos dos valores $L$ com as doses de adubo fosfatado que podem ser conseqüências dos mecanismos de mineralização e/ou imobilização, de acordo com as variações na relação C/Pinorgânico (Novais \& Smyth, 1999). Efeito semelhante do fertilizante sobre o valor $L$ foi observado por Dalal \& Hallsworth (1977).

De acordo com o conceito de valor $L$, se no sistema ocorrer a entrada de ${ }^{31} \mathrm{P}$ em formas disponíveis, dadas pela adição do fertilizante, haverá outro equilíbrio de diluição isotópica, com redução da atividade específica na planta, com conseqüente acréscimo do valor $L$, ou em outras palavras, do $\mathrm{P}$ disponível do solo. A quantidade de $\mathrm{P}$ na planta proveniente do solo (Ppps) foi significativamente diferente e crescente com a adubação. No solo LVA, na variedade Santa Cruz e tratamento não adubado, esse valor foi $2,01 \mathrm{mg} \mathrm{e}$, no tratamento adubado, foi $2,43 \mathrm{mg}$, e na variedade Santa Clara, 1,16 e 3,22 mg, sem e com adubo, respectivamente. Esse fato justifica o incremento do valor $L$ com a fertilização. Observou-se um comportamento similar no solo LV, mas com valores inferiores.

Os maiores valores $L$ no solo LVA que no solo LV são explicados pela maior extração de $P$ do solo pelas plantas em decorrência dos maiores teores de $\mathrm{Ca}^{+2} \mathrm{e} \mathrm{Mg}^{+2}$ trocáveis, $\mathrm{pH}$ levemente alcalino, e menor capacidade de sorção e força de retenção de $P$, assim como da maior quantidade de P disponível no solo LVA (Tabela 1).

Os resultados observados na literatura ainda não são categóricos em relação aos valores $L$ e $A$. Assim, Morales (1981) reportou valores constantes e independentes da aplicação de fertilizante, Volk \& McLean (1963) verificaram incrementos do valor $L$ com o aumento da dose de $P$, enquanto Nye \& Foster (1958) encontraram diminuição do valor $L$ com doses crescentes de $P$ no solo.

$O$ valor $A$ que avalia a quantidade de $P$ disponível no solo considerando a relação entre \%Ppps e \%Pppf e dose de fertilizante aplicada, não permite sua determinação sem a adição de fertilizante e não discrimina as mudanças na variação do $P$ disponível do solo com a mesma sensibilidade que o valor $L$ (Neptune \& Muraoka, 1978; Fardeau et al., 1996).

\section{CONCLUSÕES}

A variedade de tomate Santa Cruz, com maiores eficiências fisiológica, ecofisiológica e agronômica em relação à nutrição fosfórica, apresenta potencial para a utilização em planos de melhoramento genético, visando a obtenção de cultivares eficientes em solos com baixa disponibilidade de P. 


\section{REFERÊNCIAS BIBLIOGRÁFICAS}

BOWEN, G.D.; ZAPATA, F. Efficiency in uptake and use of nitrogen by plants. In: INTERNATIONAL SYMPOSIUM ON THE USE OF STABLE ISOTOPES IN PLANT NUTRITION, SOIL FERTILITY AND ENVIRONMENTAL STUDIES, Vienna, 1990. Proceedings. Vienna: IAEA, 1991. p.349-362.

COLTMAN, R.R.; GERLOFF, G.C.; GABELMAN, W.H. Differential tolerance of tomato strains to maintained and deficient levels of phosphorus. Journal of American Society for Horticultural Science, v.110, p.140-144, 1985.

DALAL, R.C.; HALLSWORTH, E.G. Measurement of isotopic exchangeable soil phosphorus and interrelationship among parameters of quantity, intensity and capacity factors. Soil Science Society of the America Journal, v.41, p.541-546, 1977.

FARDEAU, J.C.; GUIRAUD, G.; MOREL, C. The role of isotopic techniques on the evaluation of the agronomic effectiveness of $P$ fertilizers. Fertilizer Research, v.45, p.101-109, 1996.

FNP CONSULTORIA E COMÉRCIO. Agrianual 2000. São Paulo, 2000. 545p.

HORTS, W.J.; WIESLER F. Genotypic differences in acquisition and utilization of phosphorus in wheat. In: INTERNATIONAL ATOMIC ENERGY AGENCY. Isotope studies on plant productivity. Vienna: IAEA, 1996. p.73-87. (TECDOC, 889)

JACKSON, M.L. Soil chemical analysis. Englewood Cliffs: Prentice-Hall, 1958. 498p.

LEAL, N.R. Comparação da produtividade do cultivar de tomate Alcobaça com três cultivares do tipo Santa Cruz, na Baixada Fluminense. Revista Ceres, v.20, p.65-67, 1973.

MORALES, L.E.M. Fatores que afetam a determinação do fósforo isotopicamente trocável em solos. Piracicaba, 1981. 82p. Dissertação (Mestrado) - Centro de Energia Nuclear na Agricultura, Universidade de São Paulo.

MURAOKA, T. Uso de técnicas isotópicas nos estudos da fertilidade do solo. In: OLIVEIRA, A.J.; GARRIDO, W.E.; ARAÚJO, J.D.; LOURENÇO, S. (Coord.) Métodos de pesquisa em fertilidade do solo. Brasília: EMBRAPA, SEA, 1991. p.255-273.

NEPTUNE, A.M.L.; MURAOKA, T. Uso de isótopos em química e fertilidade do solo. Revista Brasileira de Ciência de Solo, v.2, p.151-163, 1978.

NOVAIS, R.F.; SMYTH, T.J. Fósforo em solo e planta em condições tropicais. Viçosa: UFV, Departamento de Solos, 1999. 399p.
NYE, P.H.; FOSTER, W.N.M. A study of the mechanism of soilphosphate uptake in relation to plant species. Plant and Soil, v.9, p.338-352, 1958.

OLSEN, S.R.; SOMMERS, L.E. Phosphorus. In: PAGE, A.L.; MILLER, R.H.; KEENEY, D.R. (ED.) Methods of soils analysis. 2.ed. Madison: ASA; SSSA, 1982. pt.2, p.403-430.

PEIXOTO, J.R.; OLIVEIRA, C.M.; SILVA, R.P.; ANGELIS, B.; CECÍLIO FILHO, B.A. Avaliação de genótipos de tomateiro tipo santa cruz no período de inverno, em Araguari, MG. Pesquisa Agropecuária Brasileira, v.34, p.2247-2251, 1999.

POMBO, G.I.; SMITH, C.B. Growth and nutrient interrelationships of three vegetable crops with different sensitivities to soil pH as affected by lime and fertilizer treatments. Communications in Soil Science and Plant Analysis, v.17, p.353-368, 1986.

RACHINSKII, V.V. Curso básico de física atómica aplicada. Moscú: Academia Agrícola K.A. Timiriazev, 1974. 391p.

RAIJ, B. van; QUAGGIO, J.A.; CANTARELLA, H.; FERREIRA, M.E.; LOPES, A.S.; BATAGLIA, O.C. Análise química do solo para fins de fertilidade. Campinas: Fundação Cargill, 1987. 170p.

SILVA, A.E.; GABELMAN, W.H. Screening maize inbred lines for tolerance to low-P stress condition. Plant and Soil, v.146, p.181-187, 1992.

SILVA, J.T.A.; BRAGA, J.M. Influência de características físicas e químicas sobre o fator capacidade-tampão de fósforo em Latossolos do Estado de Minas Gerais. Revista Ceres, v.41, p.575-583, 1993.

SILVA, R.P. Avaliação de genótipos de tomate tipo Santa Cruz, no período de verão em Araguari - MG. Uberlândia: Universidade Federal de Uberlândia, 1996. 31p.

SIMON, L.; SMALLEY, T.J.; JONES JR., J.B.; LASSEIGNE, F.T. Aluminum toxicity in tomato. 1. Growth and mineral nutrition. Journal of Plant Nutrition, v.17, p.293-306, 1994.

SMILLIE, G.W.; SYERS, J.K. Calcium fluoride formation during extraction of calcareous soil with fluoride: II. Implications to the Bray-1 test. Soil Science Society of America Proceedings, v.36, p.25-30, 1972.

VOLK, V.V.; MCLEAN, E.O. The fate of applied phosphorus in four Ohio soils. Soil Science Society of America Proceedings, v.27, p.53-58, 1963.

$\overline{\text { Recebido em } 07.11 .00}$ 